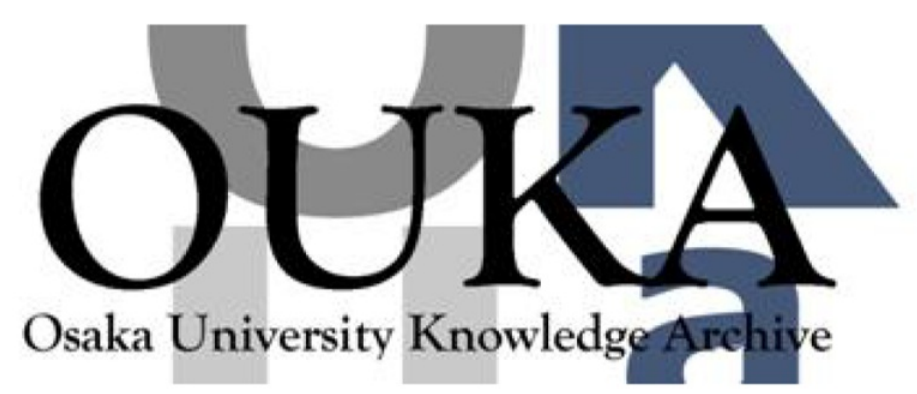

\begin{tabular}{|c|c|}
\hline Title & $\begin{array}{l}\text { MEMS hydrogen gas sensor with wireless quartz } \\
\text { crystal resonator }\end{array}$ \\
\hline Author (s) & $\begin{array}{l}\text { Zhou, Lianjie; Kato, Fumihito; Nakamura, } \\
\text { Nobutomo et al. }\end{array}$ \\
\hline Citation & $\begin{array}{l}\text { Sensors and Actuators, B: Chemical. } 334 \\
\text { p. } 129651\end{array}$ \\
\hline Issue Date & $2021-02-16$ \\
\hline oaire:version & AM \\
\hline URL & https://hdl. handle. net/11094/84198 \\
\hline rights & $\begin{array}{l}\text { (- } 2021 \text { Elsevier B.V. This manuscript version is } \\
\text { made avai lable under the Creative Commons } \\
\text { Attribution-NonCommercial-NoDerivatives } 4.0 \\
\text { International License. }\end{array}$ \\
\hline Note & \\
\hline
\end{tabular}

Osaka University Knowledge Archive : OUKA

https://ir. Library. osaka-u. ac. jp/

Osaka University 


\title{
MEMS hydrogen gas sensor with wireless quartz crystal resonator
}

\author{
Lianjie Zhou ${ }^{a}$, Fumihito Kato $^{b}$, Nobutomo Nakamura ${ }^{c}$, Yasushi Oshikane ${ }^{a}$, \\ Akira Nagakubo ${ }^{a}$, and Hirotsugu Ogi ${ }^{a *}$ \\ ${ }^{a}$ Graduate School of Engineering, Osaka University \\ Yamadaoka 2-1, Suita, Osaka 565-0871, Japan \\ ${ }^{b}$ Department of Mechanical Engineering, \\ Nippon Institute of Technology, 4-1 Gakuendai, Miyashiro-machi, Minamisaitama, \\ Saitama, 345-8501, Japan \\ ${ }^{c}$ Graduate School of Engineering Science, Osaka University \\ Toyonaka, Osaka 560-8531, Japan
}

\begin{abstract}
A highly sensitive hydrogen-gas sensor fabricated using MEMS technology is presented. The sensor chip consists of glass substrates, silicon substrate, and an AT-cut quartz crystal resonator, which is embedded in the microchannel constructed on the substrates. The quartz resonator has a fundamental resonant frequency of $165 \mathrm{MHz}$ and a $200 \mathrm{~nm}$ palladium film deposited on its single surface as the hydrogen-gas sensing material. The MEMS hydrogen-gas sensor operates in a wireless manner by exciting and detecting the resonator vibration using the non-contacting antennas. The curvature induced resonant frequency change of the resonator plate caused by the expansion of the palladium film is used for the detection of the hydrogen gas. We succeeded in improving the hydrogen absorption rate and then the sensitivity for the hydrogen-gas detection by applying the air-plasma treatment method, and clarified the role of palladium oxide in lowering the energy barrier for the hydrogen-atom migration from surface to subsurface with the X-ray photoelectron spectroscopy. Thus sensitivity enhanced MEMS hydrogen-gas sensor exhibits a detection limit of $10 \mathrm{ppm}$ or less at room temperature both in nitrogen and air.
\end{abstract}

Keywords: QCM, hydrogen-gas sensor, MEMS, wireless, air-plasma, surface oxidation 


\section{Introduction}

The development of sensitive, reliable, and low-cost hydrogen-gas sensor is of great importance with consideration of the widespread usage of hydrogen gas as a clean and sustainable energy carrier in the short future. In the recent decades, various types of hydrogen-gas sensors have been developed, including acoustical[1, 2], optical[3], catalytic[4], electrochemical[5] methods and so on. Hydrogen-gas sensors based on palladium and its alloys rely on the changes in volume or electrical impedance upon their selective hydrogen absorption ability[6-11]. For the hydrogen-gas sensors using discontinuous palladium film, the nanogaps between micropillars[8] or nanoclusters[11] must be controlled precisely. In the ball surface acoustic wave (SAW) hydrogen-gas sensor, the optimum propagation path has to be precisely searched because of the crystallographic anisotropy, which is a time-consuming procedure, and temperature compensation is also necessary because of low temperature stability of the SAW $[12,13]$. In addition to palladium, metal oxides like $\mathrm{ZnO}[14-16]$, $\mathrm{TiO}_{2}[17]$, and $\mathrm{SnO}_{2}[18]$, are used as the sensing materials. However, they invariably need to be heated up to $\sim 150{ }^{\circ} \mathrm{C}-900{ }^{\circ} \mathrm{C}[19]$. The metal oxide based semiconductor type hydrogen-gas sensors have been widely used for detecting hydrogen gas, but they require oxygen for stable operation and cannot be used in a vacuum or in spaces filled with inert gases such as fuel battery plants[20]. Also, they should not be used in a high-concentration hydrogen-gas environment for safety. A sensitive hydrogen-gas sensor that works at room temperature without temperature controlling is then preferable, especially for long term monitoring applications. In our previous study, we demonstrated that a quartz resonator coated with a palladium thin film can be used as a highly sensitive hydrogen-gas sensor[21, 22]. The absorption of hydrogen leads to the palladium-film expansion and then the geometry change of the quartz resonator, resulting in the resonant-frequency change. However, we needed to increase the resonator temperature up to $55^{\circ} \mathrm{C}$. Furthermore, we needed to manually set the sensor chip in the flow channel and to flow the sample gas; such measurement configuration deteriorates the practicalness.

In this paper, we present a wireless hydrogen-gas sensor with a thin quartz-crystal resonator embedded in a microchannel fabricated using microelectromechanical-systems (MEMS) technology. The MEMS sensor chip has advantages of a compact size, low-cost, reusable, and low power-consumption, hence it is suitable for portable and in situ hydrogen-gas detection. Moreover, 
the hydrogen-gas sensor works in a wireless-electrodeless manner, in which, the resonator excitation and signal readout are contactlessly performed by antennas; no mechanical contact is needed to the sensing area, allowing a high-Q value $(\sim 80,000)$. Also, this avoids many safety concerns considering the highly flammable and explosive properties of hydrogen gas. We intend to detect hydrogen gas through the frequency change caused by the resonator bending induced by hydrogen absorption, because this mechanism is more dominant than the mass loading effect[21]. Therefore, free deformation of the resonator should be realized, but existing quartz-crystal-microbalance sensors fail to achieve this, because quartz resonators there are tightly fixed. On the other hand, in our MEMS sensor developed in this study, a very thin quartz resonator is located in the microchannel without fixed and clamped parts, allowing nearly free bending deformation of the resonator. Since the MEMS hydrogen-gas sensor we propose here shows high sensitivity without oxygen, it can be deployed not just for detecting hydrogen-gas leakage in air but also in nitrogen-purged or inert-gas environment, such as reactor vessels, catalyst units, fuel batteries, and so on.

Experimental and theoretical studies[23-26] have proven that a subsurface layer exists in palladium film, which acts as an intermediate between chemisorbed and dissolved hydrogen atoms. Accordingly, the hydrogen absorption into palladium proceeds in three steps[27]: (i) Dissociative chemisorption on palladium surface, (ii) migration from surface to subsurface, and (iii) bulk diffusion inside palladium lattice. The activation energy for hydrogenatom migration from surface to subsurface is around $0.4 \mathrm{eV}[26]$, which is higher than that of bulk diffusion $(\sim 0.23 \mathrm{eV}[28])$. Consequently, for a palladium film, the overall absorption rate is more likely to be limited by the second step. In the work by Ward and Dao[27], it was indicated that the bulk diffusion was the rate-limiting step for palladium membranes thicker than $1 \mu \mathrm{m}$ at moderately high temperatures. Other critical thickness, such as $10 \mu \mathrm{m}$ and $20 \mu \mathrm{m}$ have also been reported[29, 30]. In our previous study, however, the evaluation of reaction constant performed on a $200 \mathrm{~nm}$ thick palladium film yielded an activation energy of $0.372 \mathrm{eV}[21]$, indicating that the surface-to-subsurface transition is the rate-limiting step in this thickness.

For making a sensitive resonator for room-temperature use, we propose the surface oxidation procedure on the palladium film using the air-plasma. We have previously observed remarkable increase in the reaction constant of hydrogen absorption into palladium film after the air-plasma treatment[22]. In this study, we investigate its mechanism by studying the hydrogen absorp- 
tion kinetics using the MEMS hydrogen-gas sensor after the plasma treatment, and find that the activation energy is significantly lowered. We also study the surface oxidation effect using the X-ray photoelectron spectroscopy (XPS) to confirm the subsurface oxygen atoms, which decrease the energy barrier and promote the surface-to-subsurface migration of hydrogen atoms. After enhancement by the air-plasma treatment, the MEMS hydrogen-gas sensor with $165-\mathrm{MHz}$ resonator shows high sensitivity, being able to detect hydrogen gas with concentration as low as 10 ppm or less even at room temperature $\left(25^{\circ} \mathrm{C}\right)$. Furthermore, we investigate the sensor performance in air and the effect of relative humidity on the sensitivity for the use in broader applications.

\section{Sensor fabrication and experiment}

\subsection{Fabrication of MEMS hydrogen-gas sensor}

Figure 1 shows the components of the hydrogen-gas sensor fabricated using a MEMS process similar to that we developed before [31, 32]. The bottom glass substrate was pre-bonded to a silicon layer. The microchannel constructed on the silicon layer and upper glass substrate is $70 \mu \mathrm{m}$ in depth. An exposure window was constructed in the upper glass substrate. The ATcut quartz resonator has an in plane area of $1.8 \times 1.6 \mathrm{~mm}^{2}$. The thickness is $13.5 \mu \mathrm{m}$ or $10 \mu \mathrm{m}$, corresponding to the fundamental thickness-shear resonant frequency of 125 or $165 \mathrm{MHz}$, respectively. Using the radio frequency (RF) magnetron-sputtering method, we deposited 5-nm chromium and then 200-nm palladium films on one side surface of the resonator as the bonding layer and the hydrogen sensing material, respectively. The quartz resonator is located between the glass and silicon micropillars. Because the resonator thickness is smaller than the gap between micropillars, the resonator deformation is not restricted. After inserting the quartz resonator, the sensor chip was packaged using the anodic bonding method. Finally, the air-plasma treatment was performed for 2 minutes using a plasma cleaner (PDC-001, Harrick Plasma, $115 \mathrm{~V}, 30 \mathrm{~W}$ ) through the top window of $1.4 \mathrm{~mm}$ in diameter. The size of the rectangular microchannel is $2.1 \mathrm{~mm}$ in length and 3.2 $\mathrm{mm}$ in width. It is worth noting that although the above describes the fabrication process of a single sensor chip, we have succeeded fabricating many sensors simultaneously from a single set of silicon and glass wafers. 


\subsection{Effect of plasma treatment on hydrogen absorption kinetics}

We first investigated the influence of the plasma treatment on the energy barrier for hydrogen migration using the 125-MHz MEMS sensor by measuring the absorption behavior at various temperatures. The MEMS sensor chip treated by the air plasma was set into the homebuilt sensor room, which was placed on a heater to maintain a desired temperature. The hydrogengas concentration in air is about $0.55 \mathrm{ppm}$ and varies with regions[33]. To accurately evaluate the sensitivity of the hydrogen-gas sensor and especially the absorption kinetics, we used a high purity nitrogen gas instead of air as the carrier gas. The sensor chip was exposed to a $99.9999 \%(6 \mathrm{~N})$ nitrogen-gas flow, and we then injected the sample gas, which was prepared by mixing the $6 \mathrm{~N}$ nitrogen gas and 1,100-ppm hydrogen gas. The hydrogen concentration in the sample gas was controlled by changing the mixing ratio. The excitation of the resonator vibration and the signal readout were performed by the antennas located outside the microchannel (Fig. 1). The resonant frequency was monitored using a network analyzer (ZNLE3, Rohde \& Schwarz).

\subsection{XPS characterisation of palladium surface}

The chemical state of palladium surface with and without the air-plasma treatment was investigated by XPS. The XPS experiments were carried out using a photoelectron spectrometer (Quantera SXM ${ }^{T M}$, ULVAC-PHI, Inc., Kanagawa, Japan). The spectra were acquired using the $\mathrm{Al} K_{\alpha} \mathrm{X}$-ray source $(h \nu=1486.6 \mathrm{eV})$. The take-off angle of photoelectron was $45^{\circ}$.

\subsection{Room temperature hydrogen-gas detection with 165-MHz MEMS sensor}

The hydrogen-gas detection at room temperature $\left(25^{\circ} \mathrm{C}\right)$ was performed using the MEMS sensor chip packaged with the $165-\mathrm{MHz}$ resonator. The sensor chip was exposed to the sample gasses with hydrogen gas of 1-250 ppm in the nitrogen gas and synthetic air $\left(\mathrm{N}_{2} 78-80 \%, \mathrm{O}_{2} 20-22 \%, \mathrm{C}_{n} \mathrm{H}_{m}\right.$, $\left.\mathrm{CO}, \mathrm{CO}_{2}<0.1 \mathrm{ppm}\right)$, respectively. The relative humidity $(\mathrm{RH})$ of the synthetic air is less than $\sim 0.01 \%$. To evaluate the effect of humidity on the performance of the hydrogen-gas sensor, we prepared hydrogen gases with different humidity by mixing the synthetic air with the hydrogen gas from a sample bag filled with ultrapure water. The actual relative humidity was measured by a humidity meter placed at the outlet of the sensor room. We monitored the transmission signal (S12) using the network analyzer during exposure. The temperature coefficient of an AT-cut quartz resonator around room temperature is of the order of $10^{-7} / \mathrm{K}$ or less [34], and the frequency 
change caused by the temperature variation during the measurement can be ignored compared with the frequency change due to the gas adsorption: The quartz resonator shows excellent frequency stability even without controlling temperature. We thus did not control the temperature during the measurement.

\section{Results and discussion}

\subsection{Hydrogen absorption kinetics on plasma treated palladium film}

Figure 2 shows the frequency response of the MEMS sensor when exposed to the hydrogen gases with different concentrations between 50 and $250 \mathrm{ppm}$ at various temperatures. The frequency drop is observed upon the hydrogen-gas injection, and after this drop, the resonant frequency nearly linearly decreases. This slope becomes larger as the hydrogen-gas concentration increases. After replacing the sample gas by the $6 \mathrm{~N}$ nitrogen gas, the resonant frequency recovers.

Figure 3 illustrates the frequency decreases during exposure to hydrogen gas. When hydrogen is adsorbed in the palladium film, it expands and causes bending deformation of the resonator. We previously found that the increase in the resonator curvature results in the decrease in the resonance frequency for an AT-cut quartz plate[21]. Therefore, the frequency decrease during exposure to hydrogen gas indicates the progress of the absorption reaction of hydrogen in the palladium film. Owing to higher diffusion speed of hydrogen atoms in palladium at higher temperature, faster rate of frequency decrease can be observed accordingly.

The frequency drop at the injection, which will be attributed to fast attachment of hydrogen gas on the palladium surface, becomes insignificant at elevated temperatures. This indicates that hydrogen-gas molecules on palladium surface is more stable at lower temperatures as shown previously[35]. However, because slightly high gas pressure in our injector also causes the transient frequency change as shown in measurement of the nitrogen-gas injection in Fig. 3, it is difficult to extract the hydrogen-attachment contribution at present. We, therefore, use the linear frequency change after the transient drop at the injection in the evaluation of the reaction kinetics.

The frequency response caused by the curvature change of an AT-cut quartz resonator during hydrogen-gas exposure can be described as[21],

$$
\Delta f(t) / f_{0}=-A\left(k_{a} C_{H_{2}}+k_{d}\right) \cdot t
$$


where $C_{H_{2}}$ denotes the concentration of hydrogen gas, $k_{a}$ and $k_{d}$ represent the reaction-velocity constants for hydrogen absorption and discharge reactions, respectively. Figure 4(a) shows the relationship between the slope of frequency decrease and the hydrogen-gas concentration, showing good linearity at various temperatures. The reaction-velocity constant $k_{a}$, which represents the absorption rate follows the Arrhenius law as,

$$
k_{a}=A_{0} \cdot \exp \left(-E / k_{B} T\right)
$$

here, $A_{0}$ is a pre-exponential factor, and $E$ is the activation energy for hydrogen atom migration. The reaction-velocity constant $k_{a}$ can be calculated as the slope of the frequency slope versus hydrogen concentration according to Eq.(1). Figure 4(b) plots the value of $k_{a}$ at different temperatures, in which, the Arrhenius fitting of logarithmical $k_{a}$ to $1 / T$ yields the activation energy of $0.238 \pm 0.024 \mathrm{eV}$. Therefore, the air-plasma treated palladium film exhibits significantly smaller energy barrier for the hydrogen atom migration from surface to subsurface compared with the palladium film without the air-plasma treatment in our previous study $(0.372 \mathrm{eV}[21])$.

\subsection{XPS characterisation of palladium surface oxidation by air-plasma}

Figure 5 represents the $\mathrm{Pd} 3 \mathrm{~d}$ and $\mathrm{Pd} 3 \mathrm{p}$ core level spectra of as-deposited and air-plasma treated palladium films. In Fig. 5(a), the Pd 3d spectrum of as deposited palladium film shows a doublet with binding energies at 334.16 $\mathrm{eV}$ for the $\mathrm{Pd} 3 \mathrm{~d}_{5 / 2}$ component and at $339.45 \mathrm{eV}$ for the $\mathrm{Pd} 3 \mathrm{~d}_{3 / 2}$ component. The atomic oxygen at palladium surface and subsurface can be detected as a binding energy shift of palladium core level because of the bond to oxygen atoms[36]. For the palladium film treated by the air plasma, both peaks of $\mathrm{Pd} 3 \mathrm{~d}$ core level shift to higher binding energy by $0.65 \mathrm{eV}$. This energy shift is in good agreement with that between palladium and palladium oxide reported previously (0.6 eV[37], $0.62 \mathrm{eV}[38,39])$.

Figure $5(\mathrm{~b})$ shows the core level spectra of $\mathrm{Pd} 3 \mathrm{p}_{3 / 2}$. The spectra were decomposed using a combination of Gauss and Lorentz functions with a linear background. The peaks at binding energy $531.07 \mathrm{eV}$ correspond to palladium metal. After the air-plasma treatment, a new peak appears at $530.09 \mathrm{eV}$, which is a characteristic peak of $\mathrm{O}$ 1s core level (528.92-530.38 eV[38], 529.2$530.2 \mathrm{eV}[40]$, and $\sim 530 \mathrm{eV}[41])$. In addition, a shift of the palladium peak to higher binding energy can be observed because of the existence of palladium oxide[41]. 
It is reported that surface oxidation of palladium and its alloys by heat treatment in air improved the hydrogen absorption rate[42-46], and this was explained by the increase in the surface roughness. Our results here, however, indicates that that oxygen atoms in the surface and subsurface layer of palladium significantly decrease the energy barrier for hydrogen atom migration from surface to subsurface: Increase in the effective surface will increase the amount of adsorbed hydrogen atoms, but it fails to affect the kinetics of absorption. Indeed, recent theoretical studies [47, 48], report the decrease in the energy barrier of hydrogen diffusion into subsurface by the presence of other gas species, including oxygen, on Pd surface, supporting our experimental result.

We performed the 10-min plasma treatment (longer than that performed in the sensing experiments) in the XPS measurement in order to clearly identify the effect of the plasma treatment on the chemical states on the $\mathrm{Pd}$ surface. In addition, our previous study [22] confirmed that after about 10 min plasma treatment, the palladium film showed significant improvement in the hydrogen-gas absorption ability. However, we find the that the palladium film peels off from the quartz surface for a long-time use when it was exposed to a longer plasma treatment. Therefore, we performed 2 min plasma treatment on the MEMS sensor for the stable and long-term hydrogen-gas detection in this study.

\subsection{Room temperature hydrogen-gas detection with 165-MHz MEMS sensor}

The enhancement effect of the air-plasma treatment on hydrogen absorption rate was demonstrated and the mechanism was thus clarified, and we subsequently performed the hydrogen-gas detection at room temperature using the MEMS hydrogen-gas sensor packaged with a 165-MHz quartz resonator. Since the MEMS sensor detects hydrogen gas by the frequency change caused by the deformation of quartz resonator, a higher frequency resonator with thinner thickness can conduce to a higher sensitivity.

The response of a 165-MHz MEMS hydrogen-gas sensor to the exposure of hydrogen gas in nitrogen at room temperature is shown in Fig. 6. As can be seen, the sensor shows no response when nitrogen gas is injected except the initial drop, whereas the frequency decreases linearly after the initial drop during the hydrogen-gas exposure with larger descending slope for higher concentration as illustrated in Fig. 6(b). Therefore, the MEMS hydrogen-gas sensor shows excellent sensitivity even at room temperature; hydrogen gas with concentration of $10 \mathrm{ppm}$ or less is detectable. 
Figure 7 shows the response of the 165-MHz MEMS hydrogen-gas sensor to the exposure of hydrogen gas in the synthetic air at room temperature. Figure 7 (a) shows the frequency change of the gas sensor to hydrogen gas with RH $0.01 \%$, in which, responses similar to that shown in Fig. 6(a) can be observed. Although the frequency decreases after the drop when the sensor is exposed to hydrogen gas in air are smaller compared with that in nitrogen, the detection limit of $10 \mathrm{ppm}$ can be achieved as well.

Figure 7(b) shows the frequency responses of the sensor to hydrogen gas with higher humidity (24\% and $45 \%$ ). As can be observed, the frequency drop upon hydrogen gas injection is much larger, which may be caused by the attachment of water vapor to the resonator surface. However, the slope of frequency decrease is not affected very much even in such higher humidity conditions as illustrated in Fig. 7(c). The results indicate that the MEMS hydrogen-gas sensor exhibits sufficiently high sensitivity even in air with moderate humidity at room temperature.

\section{Conclusion}

We have presented here a MEMS sensor with wireless quartz crystal resonator for hydrogen-gas detection at room temperature. The decrease in resonant frequency of the resonator due to its curvature change caused by palladium film expansion was used as a measure of the hydrogen-gas concentration. The fabricated MEMS hydrogen-gas sensor chip has advantages of compact, reusable, low cost and low-power consumption, which make it suitable for portable and in-situ monitoring application. We also proposed a convenient method to remarkably improve the sensitivity of the MEMS hydrogen-gas sensor by the air plasma. We investigated the effect of the air plasma on the hydrogen absorption kinetics onto palladium film. By studying the reaction-velocity constant of hydrogen absorption at various temperatures, an activation energy of $0.238 \mathrm{eV}$ for hydrogen atom migration was obtained, which was significantly lower than that for untreated palladium film $(0.372 \mathrm{eV})$. Furthermore, we investigated the chemical state of palladium surface treated by air plasma and found that considerable amount of atomic oxygen exists in the palladium film, indicating that the surface oxidation by the air plasma can significantly lower the energy barrier of hydrogen migration from surface to subsurface. We performed hydrogen-gas detection at room temperature using a $165-\mathrm{MHz}$ MEMS sensor. Attributed to the acceleration in surface-to-subsurface transfer of hydrogen atoms by 
the air-plasma treatment, hydrogen gas with concentration as low as 10 ppm or less can be detected. For demonstrating further practical application, we investigated the effect of humidity on the sensitivity of the sensor. The MEMS hydrogen-gas sensor shows high sensitivity even in air with moderate humidity.

Because the response of the quartz resonator depends on temperature and humidity (not so strongly), it is effective to prepare the calibration curves at various temperatures and humidities to precisely determine the hydrogen-gas concentrations. We also plan to construct the multichannel MEMS resonator system for measuring temperature, humidity, and the hydrogen-gas concentration simultaneously, which will be our future study.

\section{Acknowledgment}

This research was supported by Development of Advanced Measurement and Analysis Systems from Japan Science and Technology Agency (Project No. JP-MJSN16B5). 


\section{References}

[1] I. Constantinoiu, C. Viespe, Development of Pd/TiO2 Porous Layers by Pulsed Laser Deposition for Surface Acoustic Wave $\mathrm{H}_{2}$ Gas Sensor, Nanomaterials 10 (2020) 760 .

[2] T. Tsuji, R. Mihara, T. Saito, S. Hagihara, T. Oizumi, N. Takeda, T. Ohgi, T. Yanagisawa, S. Akao, N. Nakaso, K. Yamanaka, Highly Sensitive Ball Surface Acoustic Wave Hydrogen Sensor with Porous Pd-Alloy Film, Mater. Trans. 55 (2014) 1040-1044.

[3] Y.N. Zhang, H. Peng, X. Qian, Y. Zhang, G. An, Y. Zhao, Recent advancements in optical fiber hydrogen sensors, Sens. Actuat. B 244 (2017) 393-416.

[4] E. Lee, I. Hwang, J. Cha, H. Lee, W. Lee, J. Pak, J. Lee, B. Ju, Micromachined catalytic combustible hydrogen-gas sensors, Sens. Actuat. B 153 (2011) 392-397.

[5] G. Korotcenkov, S. D. Han, J. R. Stetter, Review of electrochemical hydrogen sensors, Chem. Rev. 109 (2009) 1402-1433.

[6] L. Zhang, H. Jiang, J. Zhang, Y. Huang, J. Tian, X. Deng, X. Zhao, W. Zhang, Flexible nanofiber sensor for low-concentration hydrogen detection, Nanotechnology 31 (2019) 015504.

[7] N. Nakamura, T. Ueno, H. Ogi, Hydrogen-gas sensing at low concentrations using extremely narrow gap palladium nanoclusters prepared by resistive spectroscopy, J. Appl. Phys. 126 (2019) 225104.

[8] M. Zhao, M. H. Wong, and C. W. Ong, Achievement of controlled resistive response of nanogapped palladium film to hydrogen, Appl. Phys. Lett. 107 (2015) 033108.

[9] C. McConnell, S. N. Kanakaraj, J. Dugre, R. Malik, G. Zhang, M. R. Haase, Y.-Y. Hsieh, Y. Fang, D. Mast, V. Shanov, Hydrogen Sensors Based on Flexible Carbon Nanotube-Palladium Composite Sheets Integrated with Ripstop Fabric, ACS Omega 5 (2020) 487-497.

[10] Z. Han, J. Ren, J. Zhou, S. Zhang, Z. Zhang, L.Yang, C. Yin, Multilayer porous $\mathrm{Pd}-\mathrm{WO}_{3}$ composite thin films prepared by sol-gel process for hydrogen sensing, Int. J. Hydrog. Energy 45(2020) 7223-7233. 
[11] N. Nakamura, T. Ueno, H. Ogi, Precise control of hydrogen response of semicontinuous palladium film using piezoelectric resonance method, Appl. Phys. Lett. 114 (2019) 201901.

[12] K. Yamanaka, N. Nakaso, D. Sim, T. Fukiura, Principle and application of ball surface acoustic wave (SAW) sensor, Acoust. Sci. Technol. 30 (2009) $2-6$.

[13] T. Yanagisawa, T. Ohgi, S. Akao, N. Nakaso, Y. Tsukahara, Y. Ohara, T. Tsuji, K. Yamanaka, Meandering collimated beam of surface acoustic waves on a trigonal crystal ball, Appl. Phys. Lett. 98 (2011) 123508.

[14] J. Kim, A. Mirzaei, H. W. Kim, P. Wu, S. S. Kim, Design of supersensitive and selective ZnO-nanofiber-based sensors for $\mathrm{H}_{2}$ gas sensing by electron-beam irradiation, Sens. Actuat. B 293 (2019) 210-223.

[15] F. Rascha, V. Posticab, F. Schütta, Y. K. Mishra, A. S. Nia, M. R. Lohe, X. Feng, R. Adelung, O. Lupan, Highly selective and ultra-low power consumption metal oxide based hydrogen-gas sensor employing graphene oxide as molecular sieve, Sens. Actuat. B 320 (2020) 128363.

[16] A. Ghosh, C. Zhang, S. Ju, H. Zhang, Selective $\mathrm{H}_{2}$ sensing using lanthanum doped zinc oxide thin film: A study of temperature dependence $\mathrm{H}_{2}$ sensing effect on carrier reversal activity, J. Appl. Phys. 128 (2020) 094504 .

[17] T. Hyodo, H. Shibata, Y. Shimizu, M. Egashira, $\mathrm{H}_{2}$ sensing properties of diode-type gas sensors fabricated with Ti- and/or Nb-based materials, Sens. Actuat. B 142 (2009) 97-104.

[18] T. Hamaguchi, N. Yabuki, M. Uno, S. Yamanaka, M. Egashira, Y. Shimizu, T. Hyodo, Synthesis and $\mathrm{H}_{2}$ gas sensing properties of tin oxide nanotube arrays with various electrodes, Sens. Actuat. B 113 (2006) $852-856$.

[19] V. Aroutiounian, Metal oxide hydrogen, oxygen, and carbon monoxide sensors for hydrogen setups and cells, Int. J. Hydrogen Energy 32 (2007) $1145-1158$. 
[20] W. J. Buttner, M. B. Post, R. Burgess, C. Rivkin, An overview of hydrogen safety sensors and requirements, Int. J. Hydrog. Energy 36 (2011) $2462-2470$.

[21] L. Zhou, N. Nakamura, A. Nagakubo, H. Ogi, Highly sensitive hydrogen detection using curvature change of wireless-electrodeless quartz resonators, Appl. Phys. Lett. 115 (2019) 171901.

[22] L. Zhou, N. Nakamura, A. Nagakubo, H. Ogi, Enhancement of sensitivity of Pd-based hydrogen-gas sensor by plasma exposure studied by wireless quartz resonator, Jpn. J. Appl. Phys. 59 (2020) SKKB02.

[23] R. J. Behm, V. Penka, M.-G. Cattania, K. Christmann, G. Ertl, Evidence for "subsurface" hydrogen on $\operatorname{Pd}(110)$ : An intermediate between chemisorbed and dissolved species, J. Chem. Phys. 78 (1983) 7486-7490.

[24] H. Okuyama, W. Siga, N. Takagi, M. Nishijima, T. Aruga, Path and mechanism of hydrogen absorption at $\operatorname{Pd}(100)$, Surf. Sci. 401 (1998) 344354.

[25] K.H. Rieder, M. Baumberger, W. Stocker, Selective Transition of Chemisorbed Hydrogen to Subsurface Sites on Pd(110), Phys. Rev. Lett. 51 (1983) 1799-1802.

[26] P. Ferrin, S. Kandoi, A.U. Nilekar, M. Mavrikakis, Hydrogen adsorption, absorption and diffusion on and in transition metal surfaces: A DFT study, Surf. Sci. 606 (2012) 679-689.

[27] T.L. Ward, T. Dao, Model of hydrogen permeation behavior in palladium membranes, J. Membr. Sci. 153 (1999) 211-231.

[28] Y. Fukai, H. Sugimoto, Diffusion of hydrogen in metals, Adv. Phys. 34 (1985) 263-326.

[29] S. Uemiya, N. Sato, H. Ando, Y. Kude, T. Matsuda, E. Kikuchi, Separation of hydrogen through palladium thin film supported on a porous glass tube, J. Membr. Sci. 56 (1991) 303-313.

[30] R. C. Hurlbert, J. O. Konecny, Diffusion of Hydrogen through Palladium, J. Chem. Phys. 34 (1961) 655-658. 
[31] F. Kato, H. Ogi, T. Yanagida, S. Nishikawa, M. Hirao, M. Nishiyama, Resonance acoustic microbalance with naked-embedded quartz (RAMNE-Q) biosensor fabricated by microelectromechanical-system process, Biosens. Bioelectron. 33 (2012) 139-145.

[32] F. Kato, H. Noguchi, Y. Kodaka, N. Oshida, H. Ogi, Wireless poly(dimethylsiloxane) quartz-crystal-microbalance biosensor chip fabricated by nanoimprint lithography for micropump integration aiming at application in lab-on-a-chip, Jpn. J. Appl. Phys. 57 (2018) 07LD14.

[33] U. Schmidt, Molecular hydrogen in the atmosphere, Tellus 26 (1974) 78.

[34] R. Bechmann, Frequency-temperature-angle characteristics of AT-type resonators made of natural and synthetic quartz, Proceedings of the IRE 44 (1956) 1600.

[35] J.E. Lennard-Jones, Processes of adsorption and diffusion on solid surfaces, Trans. Faraday Soc. 28 (1932) 333-359.

[36] A.I. Titkov, A.N. Salanov, S.V. Koscheev, A.I. Boronin, Mechanisms of $\operatorname{Pd}(110)$ surface reconstruction and oxidation: XPS, LEED and TDS study, Surf. Sci. 600 (2006) 4119-4125.

[37] F.P. Leisenberger, G. Koller, M. Sock, S. Surnev, M.G. Ramsey, F.P. Netzer, B. Klötzer, K. Hayek, Surface and subsurface oxygen on Pd(111), Surf. Sci. 445 (2000) 380-393.

[38] D. Zemlyanov, B. Aszalos-Kiss, E. Kleimenov, D. Teschner, S. Zafeiratos, M. Hävecker, A. Knop-Gericke, R. Schlögl, H. Gabasch, W. Unterberger, K. Hayek, B. Klötzer, In situ XPS study of $\operatorname{Pd}\left(\begin{array}{lll}1 & 1 & 1\end{array}\right)$ oxidation. Part 1: 2D oxide formation in $10^{-3}$ mbar $\mathrm{O}_{2}$, Surf. Sci. 600 (2006) 983-994.

[39] E. Lundgren, G. Kresse, C. Klein, M. Borg, J.N. Andersen, M. De Santis, Y. Gauthier, C. Konvicka, M. Schmid, P. Varga, Two-dimensional oxide on Pd(111), Phys. Rev. Lett. 88 (2002) 246103-1.

[40] L.S. Kibis, A.I. Titkov, A.I. Stadnichenko, S.V. Koscheev, A.I. Boronin, $\mathrm{X}$-ray photoelectron spectroscopy study of $\mathrm{Pd}$ oxidation by $\mathrm{RF}$ discharge in oxygen, Appl. Surf. Sci. 255 (2009) 9248-9254. 
[41] M. Brun, A. Berthet, J.C. Bertolini, XPS, AES and Auger parameter of Pd and PdO, J. Electron Spectros. Relat. Phenom. 104 (1999) 55-60.

[42] H. Uchikawa, T. Okazaki, K. Sato, New Technique of Activating Palladium Surface for Absorption of Hydrogen or Deuterium, Jpn. J. Appl. Phys. 32 (1993) 5095-5096.

[43] D. Wang, J.D. Clewley, T.B. Flanagan, B. Balasubramaniam, K.L. Shanahan, Enhanced rates of hydrogen adsorption resulting from oxidation of Pd or internal oxidation of Pd-Al alloys, J. Alloys Compd. 298 (2000) 261-273.

[44] K. Zhang, S.K. Gade, Ø. Hatlevik, J.D. Way, A sorption rate hypothesis for the increase in $\mathrm{H}_{2}$ permeability of palladium-silver ( $\mathrm{Pd}-\mathrm{Ag}$ ) membranes caused by air oxidation, Int. J. Hydrog. Energy 37 (2012) 583-593.

[45] N. Vicinanza, I.-H. Svenum, T. Peters, R. Bredesen, H. Venvik, New insight to the effects of heat treatment in air on the permeation properties of thin Pd77\% Ag23\% membranes, Membranes 8 (2018) 92.

[46] A.L. Mejdell, H. Klette, A. Ramachandran, A. Borg, R. Bredesen, Hydrogen permeation of thin, free-standing $\mathrm{Pd} / \mathrm{Ag} 23 \%$ membranes before and after heat treatment in air, J. Membr. Sci. 307 (2008) 96-104.

[47] T.S. Liang, H.F. Kang, W. Zhong, H.T. Bian, J. Zhao, Impact of surface adsorbed gases on hydrogen diffusion into $\operatorname{Pd}(100)$ subsurface from first principles, Appl. Surf. Sci. 473 (2019) 476-485.

[48] H.T. Bian, H.F. Kang, W. Zhong, J. Zhao, T.S. Liang, Hydrogen diffusion into $\operatorname{Pd}(100)$ subsurface: Role of co-adsorbed bicomponent species on surface, Appl. Surf. Sci. 533 (2020) 147448. 


\section{Figure Caption}

Fig. 1 Structure of MEMS hydrogen-gas sensor. (a) top view; (b) crosssection view.

Fig. 2 Frequency responses of the 125-MHz MEMS hydrogen-gas sensor upon exposure to hydrogen gas at various temperatures. Three independent measurements are shown.

Fig. 3 Frequency responses of the 125-MHz MEMS hydrogen-gas sensor during exposure to hydrogen gas with different concentrations at various temperatures. The error bars indicate the standard deviations of three independent measurements.

Fig. 4 (a) Relationship between the slope of the frequency decrease and the hydrogen-gas concentration at various temperatures; (b) An Arrhenius plot between the reaction velocity constant $k_{a}$ versus $1 / T$. The data point at $0 \mathrm{ppm}$ in (a) (green circle) represents the measurement for nitrogen injection.

Fig. $5 \mathrm{Pd}$ 3d (a) and Pd 3p (b) core level spectra of palladium film with and without air-plasma treatment.

Fig. 6 (a) Frequency responses of the 165-MHz MEMS hydrogen-gas sensor during exposure to hydrogen gas in nitrogen with various concentrations at room temperature. (b) Relationship between the slope of the frequency decrease and the hydrogen-gas concentration.

Fig. 7 Frequency responses of the 165-MHz MEMS hydrogen-gas sensor during exposure to hydrogen gas in air with relative humidity of (a) $0.01 \%$ and (b) $24 \%$ and $45 \%$ at room temperature. (c) Relationship between the slope of the frequency decrease and the hydrogen-gas concentration. 

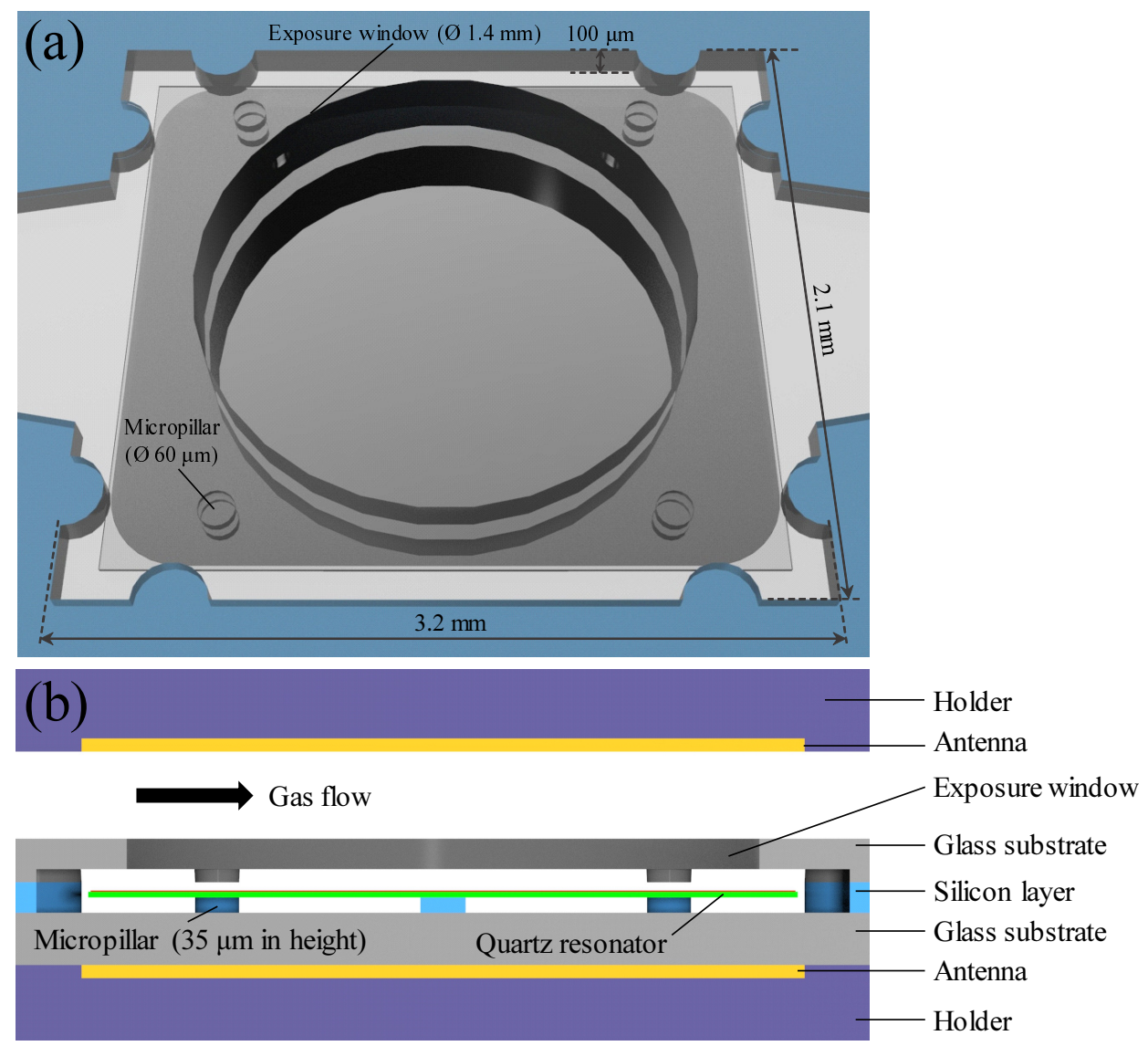

Figure 1: 


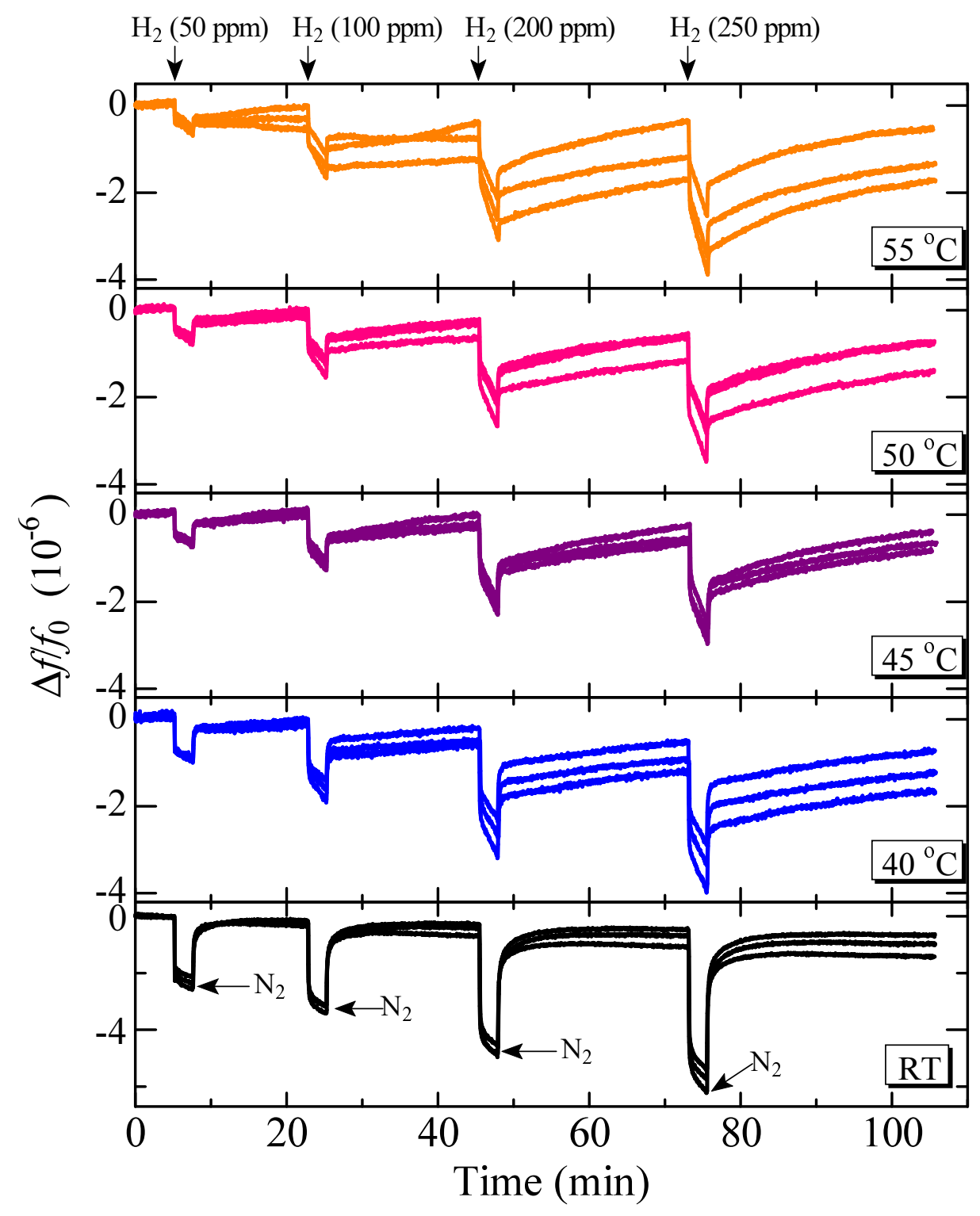

Figure 2: 

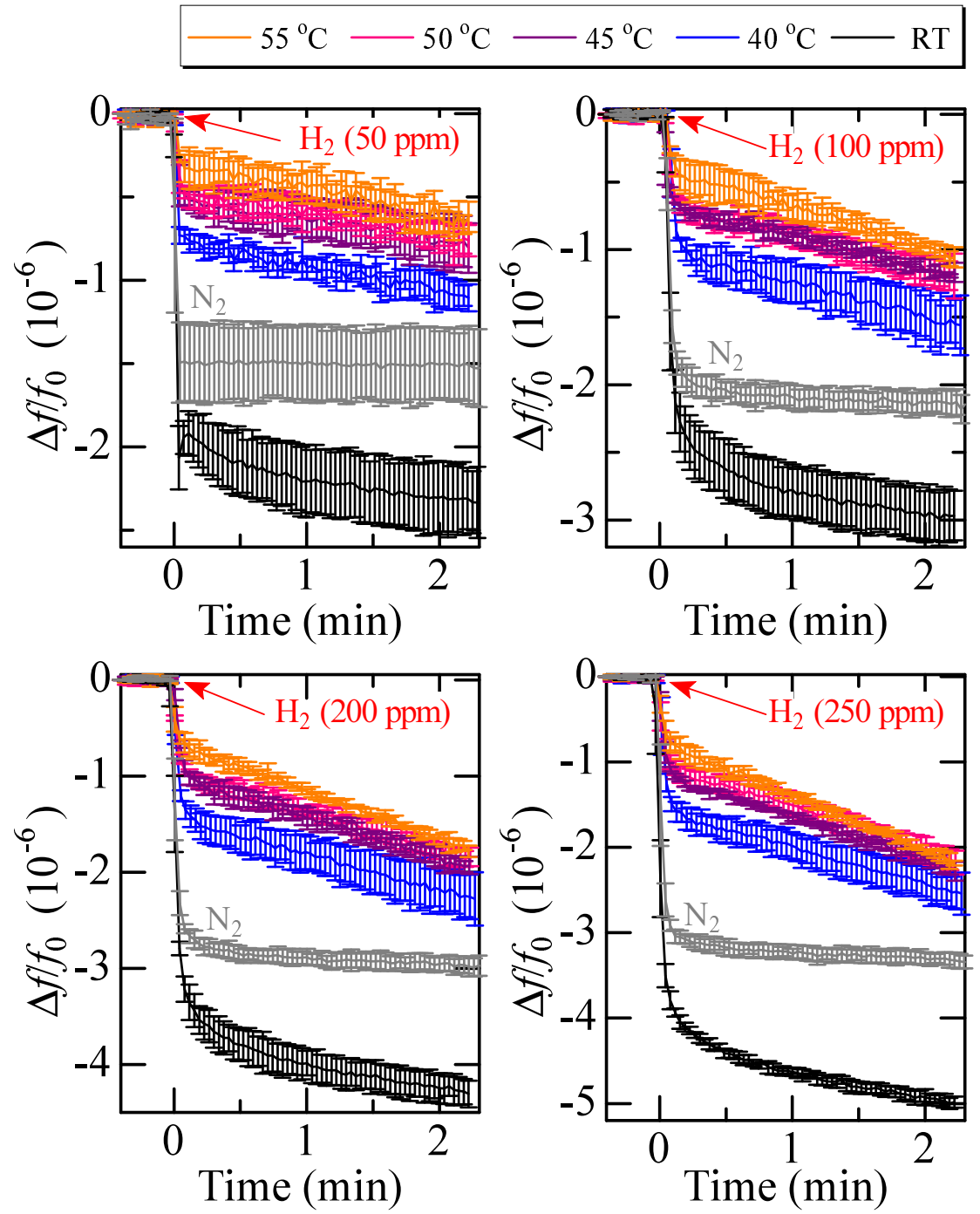

Figure 3: 

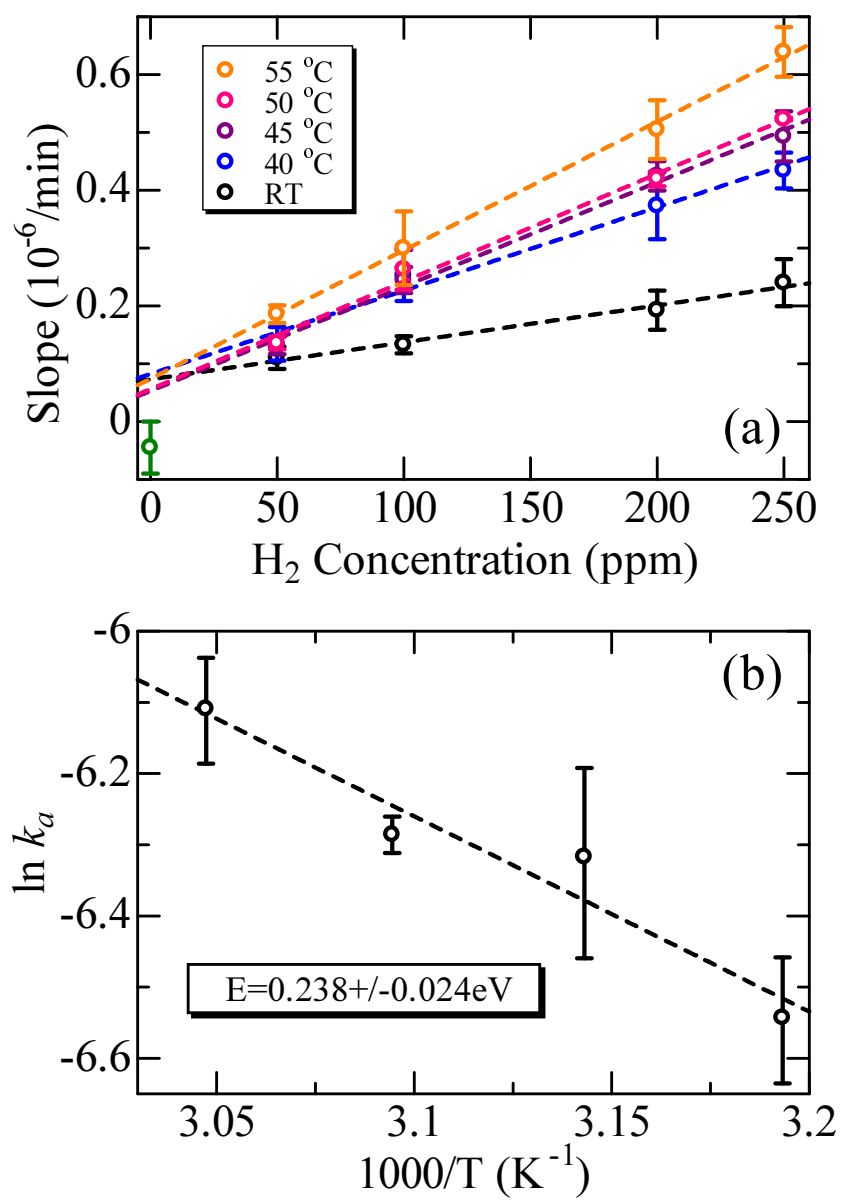

Figure 4: 

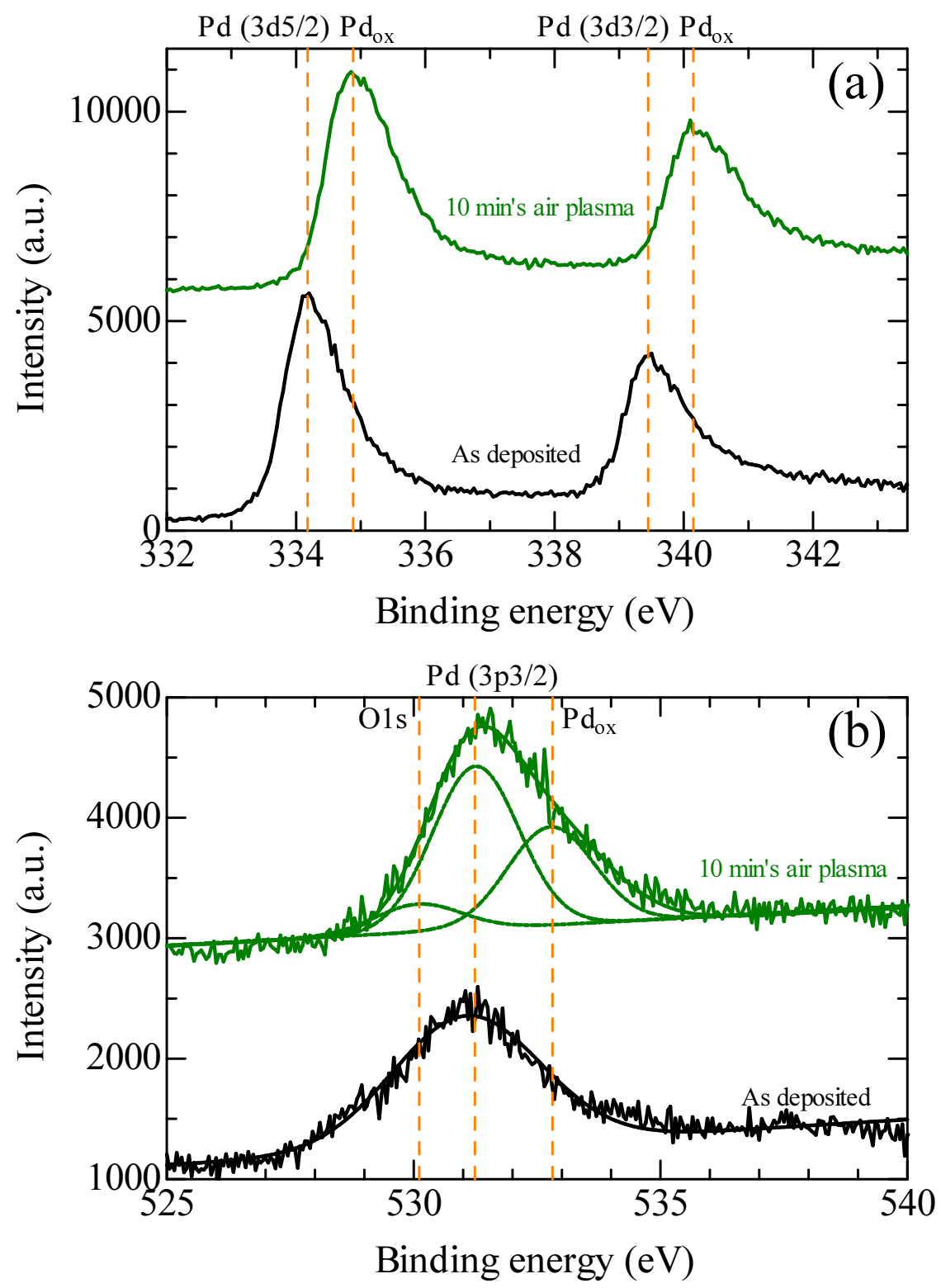

Figure 5: 

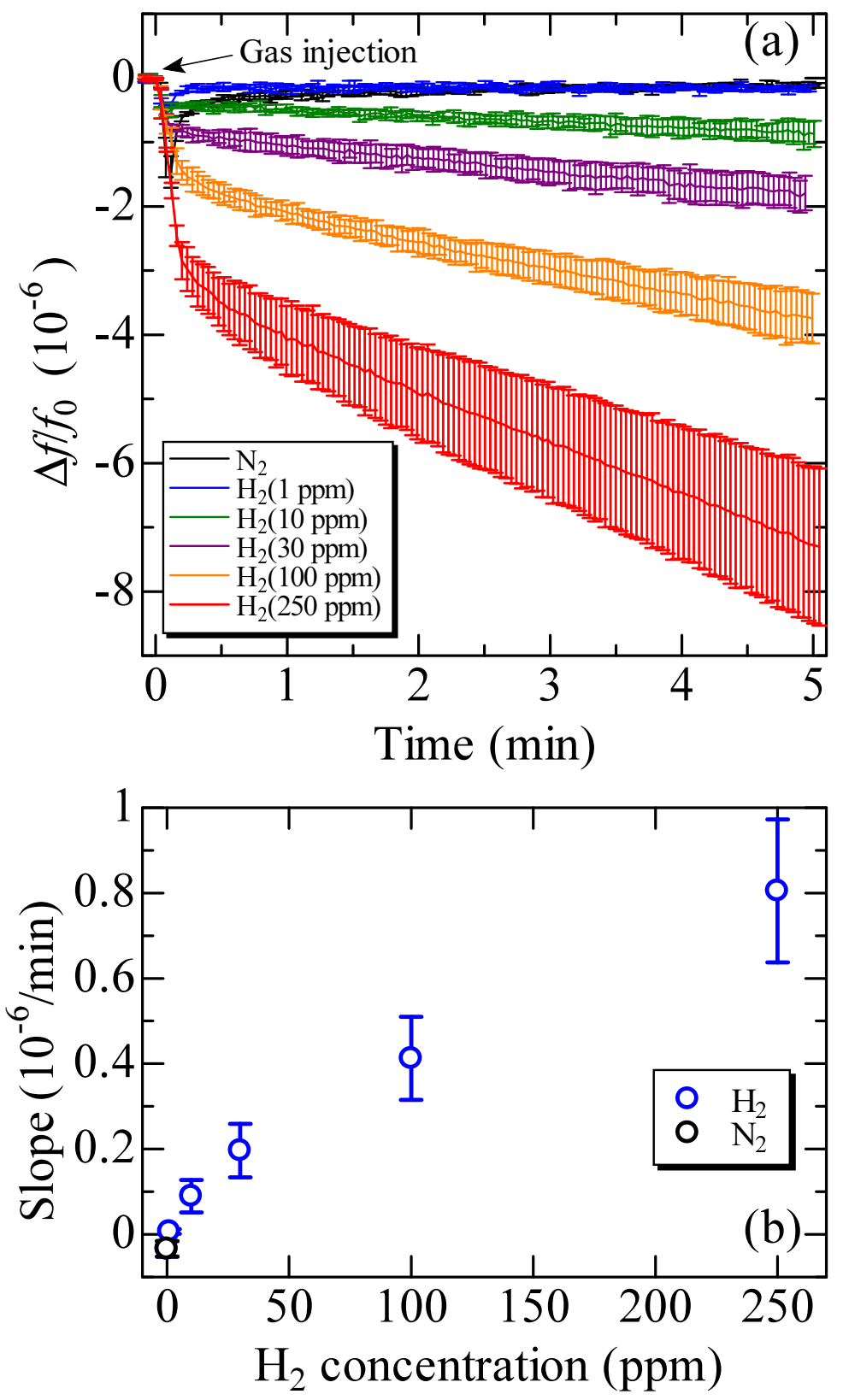

Figure 6: 

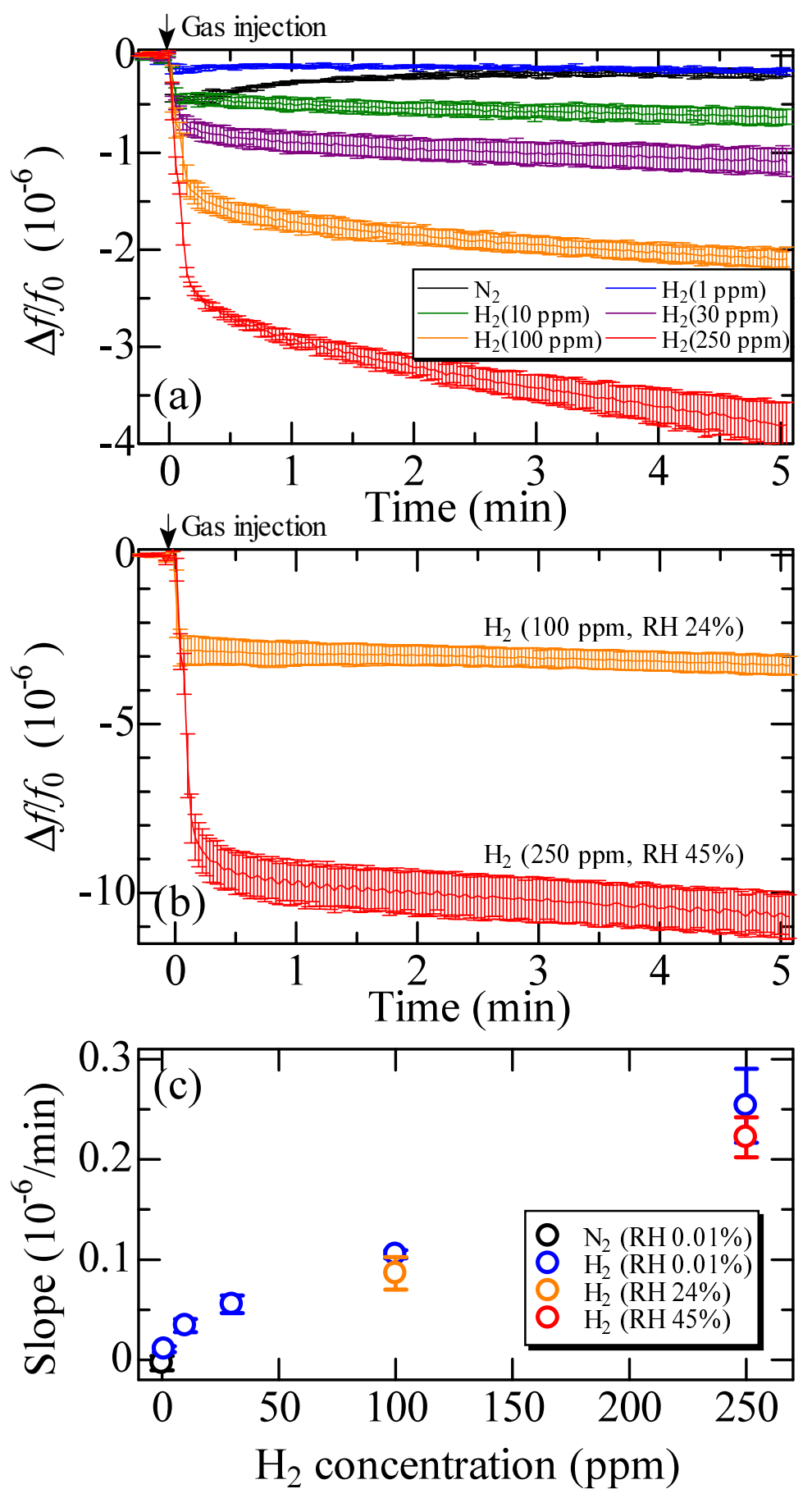

Figure 7: 

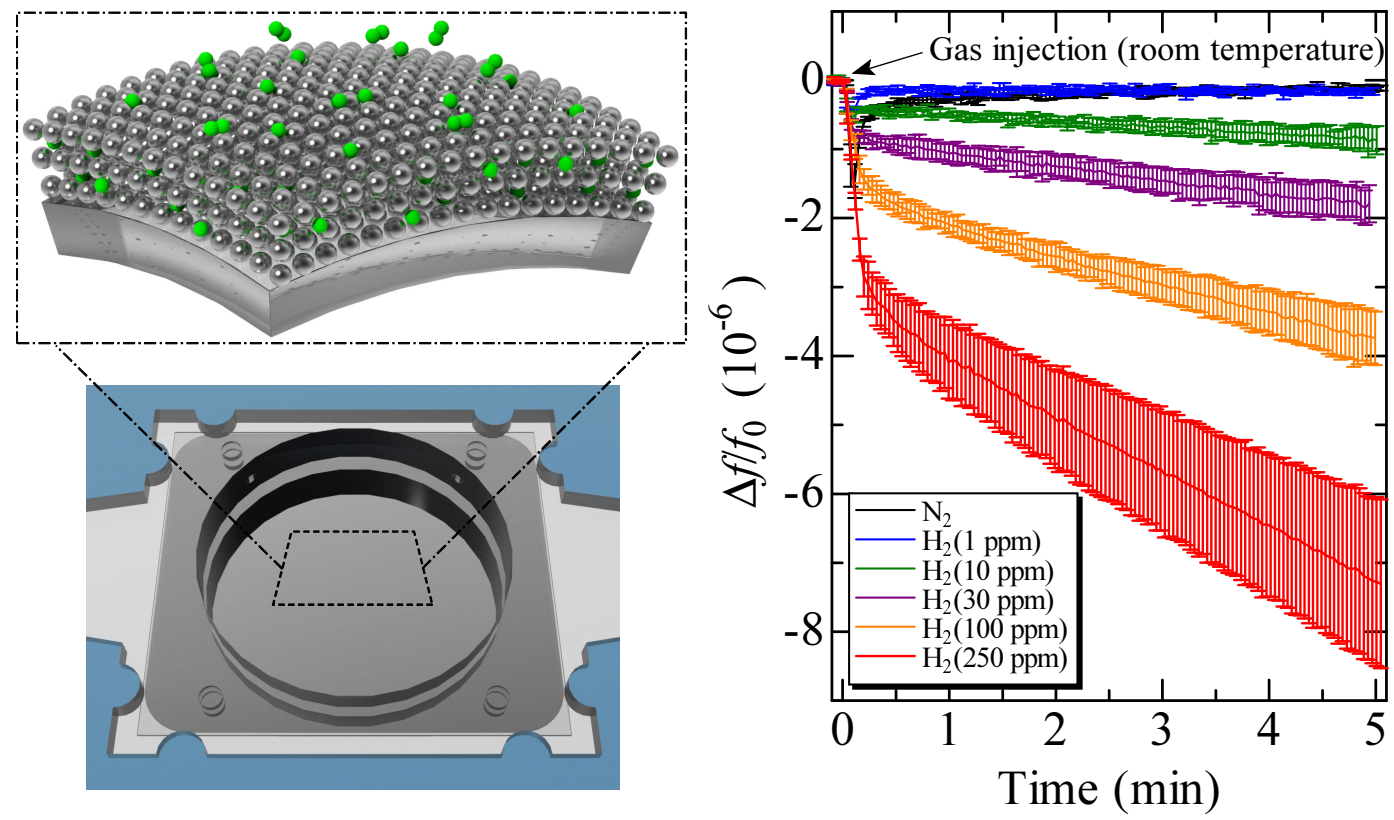

Graphical Abstract 